\title{
On the Essential Spectrum of the Transfer Operator for Expanding Markov Maps
}

\author{
Pierre Collet $^{1}$ and Stefano Isola ${ }^{2}$ \\ 1 Centre de Physique Théorique de l'Ecole Polytechnique, F-91128 Palaiseau Cédex, France \\ 2 Dipartimento di Matematica e Fisica, Università di Camerino, I-62032 Camerino, Italy
}

Received June 20, 1990; in revised form November 28, 1990

\begin{abstract}
The essential spectrum of the transfer operator for expanding markov maps of the interval is studied in detail. To this end we construct explicitly an infinite set of eigenfunctions which allows us to prove that the essential spectrum in $C^{k}$ is a disk whose radius is related to the free energy of the Liapunov exponent.
\end{abstract}

\section{Introduction}

Transfer operators have provided an important tool in the study of several questions arising from dynamical systems theory. By analogy with their use in statistical mechanics they have allowed the construction of equilibrium states (invariant measures) as well as the study of their dynamical properties (see [Bo, R1, S]). In particular, they give a very interesting connection between the Fourier transform of correlation functions for an equilibrium state and the corresponding weighted zeta function. To be slightly more precise, the large time decay of correlation functions as well as the analytic structure of the zeta function are directly connected to the spectral properties of a suitable transfer operator [R2, $\mathrm{P} 1, \mathrm{H}, \mathrm{Ec}, \mathrm{K} 2, \mathrm{Ba}$ ].

It turns out that the spectrum of these operators can be decomposed into a discrete part (i.e. the set of all isolated eigenvalues with finite multiplicity) and an essential part (cf. $[\mathrm{N}]$ ), which give contributions of very different nature to the decay of correlations. For this reason the study of the essential spectrum is a matter of growing attention [R3, R4, T, K1].

In this paper we shall study the essential spectrum for regular expanding Markov maps of the interval. Briefly, we say that a map $f$ of the interval $[0,1]$ is Markov, if there exist a finite set of disjoint open intervals $I_{1}, \ldots, I_{l}$ whose closure form a covering of $[0,1]$ and such that the closure of the image of any such interval under $f$ is again a union of such closures (such a partition is usually referred to as Markov partition). Moreover, there exist $m>0$ such that $\lambda\left(f^{-m}\left(I_{j}\right) \cap I_{i}\right) \neq 0$ for every $i, j$ (where $\lambda$ denotes the Lebesgue measure). We will assume that $f$ is 
piecewise $C^{\infty}$, i.e. its restriction to any of the above intervals $I_{1}, \ldots, I_{l}$ is $C^{\infty}$ and all the derivatives of $f$ have limits at the boundary points. We shall also assume that $f$ is expanding and regular, in the following sense:

i) there are two finite numbers $\chi \geqq \varrho>1$ such that

ii)

$$
\chi \geqq\left|f_{\mid I_{s}}^{\prime}\right| \geqq \varrho \quad \text { for } \quad s=1, \ldots, l .
$$

$$
\sup _{s} \sup _{x \in I_{s}}\left|f_{\mid I_{s}}^{\prime \prime}(x)\right| \leqq \chi
$$

It is well known that such maps have a unique absolutely continuous invariant measure (see for instance [M], pp. 167-178), whose density $\phi$ is a fixed point of the transfer operator $P$, defined for any measurable function $h$ by

$$
P h(x)=\sum_{\substack{y \\ f(y)=x}} \frac{1}{\left|f^{\prime}(y)\right|} h(y) .
$$

More generally, given any measurable function $\psi$, one can define the transfer operator $P_{\psi}$ by

It is easy to verify that

$$
P_{\psi} h(x)=\sum_{\substack{y \\ f(y)=x}} \psi(y) h(y) .
$$

and

$$
P_{\psi}^{n} h(x)=\sum_{\substack{y \\ f^{n}(y)=x}} \prod_{j=0}^{n-1} \psi\left(f^{j}(y)\right) h(y)
$$

$$
P_{\psi}(h \circ f)=h P_{\psi} 1 \text {. }
$$

A consequence of the latter property is that $P$ is dual to $f$, in the sense that

$$
\int\left(g_{1} \circ f\right) g_{2} d x=\int g_{1} P g_{2} d x
$$

whenever both sides of this equation are well defined.

It follows from our hypothesis on the transformation $f$ that the operator $P$ is bounded in $C^{k}$, and we will study its essential spectrum in this family of spaces. For the sake of simplicity we will only consider integer values of $k$, and also Markov maps $f$ satisfying

$$
\overline{f\left(I_{s}\right)}=[0,1] \text { for } s=1, \ldots, l,
$$

the case where $f$ is not full being more delicate. The arguments will be given for the case $l=2$, but they obviously extend to any value of $l$.

The main result of this paper is that under the above hypothesis, the essential spectrum of $P$ in $C^{k}$ is a disk whose radius is equal to

$$
\exp F(-k)
$$

where $F$ is the free energy of the Liapunov exponent, defined by

$$
F(\beta)=\lim _{n \rightarrow \infty} \frac{1}{n} \log \int_{0}^{1}\left|f^{n^{\prime}}(x)\right|^{\beta} \phi(x) d x .
$$

We recall that the function $F(\beta)$ is related to the large deviations (multi-fractal aspects) of the Liapunov exponent ([BPPV, B, R, C, L]). It is a convex function of $\beta$ which is $C^{1}$ and its derivative at $\beta=0$ is the Liapunov exponent of the invariant 
measure $\phi(x) d x$. Moreover, its Legendre transform is the large deviation function $[\mathrm{El}]$ for the sequence of random variables

$$
\log \left|f^{n^{\prime}}(x)\right| \text {. }
$$

Let us note finally that in the context of statistical mechanics formalism $F(\beta)$ may also be regarded as the topological pressure of the interaction function $(\beta-1) \log \left|f^{\prime}\right|$ (see [R1]). We will proceed as follows: first, we shall prove a lower bound on the essential spectral radius in $C^{0}$ for operators $P_{\psi}$. This will be obtained from the explicit construction of an infinite set of eigenfunctions. Incidentally, such a construction will provide an effective tool to investigate the actual structure of the essential spectrum, which turns out to be a disk. We will then reduce the $C^{k}$ problem to the $C^{0}$ one for a compact perturbation of the operator $P$. Finally, the upper bound will follow using a direct estimate.

\section{The Essential Spectrum in $C^{0}$}

1. Theorem. Let $\psi$ be a function whose restriction to any of the intervals of the Markov partition is $C^{\alpha}$ (with $\left.\alpha>0\right)$ and bounded away from zero. Then the essential spectrum of the operator $P_{\psi}$ in $C^{0}$ is the disk of radius $e^{F_{|\psi|}}$, where $F_{|\psi|}$ is defined by

$$
F_{|\psi|}=\lim _{n \rightarrow \infty} \frac{1}{n} \log \int_{0}^{1} \prod_{j=0}^{n-1}\left|\psi\left(f^{j}(x)\right)\right|\left|f^{n}(x)\right| \phi(x) d x .
$$

Before we give the proof we need some auxiliary results.

\section{Lemma.}

$$
\limsup _{n \rightarrow \infty} \frac{1}{n} \sup _{x \in[0,1]} \log \left|P_{\psi}^{n} 1(x)\right| \leqq \lim _{n \rightarrow \infty} \frac{1}{n} \sup _{x \in[0,1]} \log P_{|\psi|}^{n} 1(x)=F_{|\psi|},
$$

see [R1, Chap. 7], for a proof.

Remarks. (1) Note that the first limit provides an upper bound for the logarithm of the spectral radius of $P_{\psi}$.

(2) According to the above discussion, the second limit gives the topological pressure for $\log |\psi|$ (see [Bo]).

3. Lemma. For each integer $q \geqq 1, \operatorname{ker} P_{\psi}^{q}$ is infinite dimensional (the functional space $P_{\psi}$ is acting on being $\left.C^{0}\right)$.

Proof. $f^{-q}$ has $2^{q}$ monotone branches denoted by $f_{1}, \ldots, f_{2^{q}}$. They are monotone on maximal open intervals denoted respectively by $K_{1}, \ldots, K_{2 q}$. Let $r_{1}$ be a continuous function with support in the open set $K_{1}$.

Define the function $r_{j}$ (where $1 \leqq j \leqq 2^{q}$ ) by

$$
r_{j}(y)= \begin{cases}r_{1}\left(f_{1}\left(f^{q}(y)\right)\right. & \text { if } y \in K_{j} \\ 0 & \text { otherwise } .\end{cases}
$$

$r_{j}$ is obviously $C^{0}$ with support in $K_{j}$.

For any integer $l, 1 \leqq l \leqq 2^{q}-1$, we define a function $h_{q}^{l}$ by

$$
h_{q}^{l}(x)=\frac{\sum_{j=1}^{2^{q}} e^{2 \pi i(j-1) l / 2^{q}} r_{j}(x)}{\prod_{j=0}^{q-1} \psi\left(f^{j}(x)\right)} .
$$


It is straightforward to realize that these functions are linearly independent and satisfy

$$
P_{\psi}^{q} h_{q}^{l}=0
$$

Furthermore, by changing the function $r_{1}$ we can construct infinitely many such independent functions. However, since the parameter $l$ will not prove really useful in the following, we shall limit ourselves to consider the functions $h_{q} \equiv h_{q}^{l}$ where, say, $l=1$.

4. Lemma. $\exists K>0$ such that $\forall q>0$

$$
\frac{P_{|\psi|}^{q} 1(x)}{P_{|\psi|}^{q} 1(y)} \leqq K \quad \forall x, y \in[0,1] .
$$

Proof. The proof is the same as the proof of the distortion lemma. See, for instance [GH].

5. Lemma. Any point in the open disk $|z|<\inf _{x} P_{|\psi|}^{q} 1(x)$ is an infinitely degenerate eigenvalue of $P_{\psi}^{q}$ in $C^{0}$.

Proof. Let $h_{q} \in C^{0}$ such that $P_{\psi}^{q} h_{q}=0$. Let $\chi_{q}(y)$ be the sign of $\prod_{j=0}^{q-1} \psi \circ f^{j}(y)$. Then, for $z$ as above we define a function $e_{q, z}$ by

$$
e_{q, z}=\sum_{l=0}^{\infty} z^{l} \frac{\left(\prod_{j=0}^{l-1} \chi_{q} \circ f^{j q}\right) \cdot\left(h_{q} \circ f^{l q}\right)}{\prod_{j=1}^{l}\left(P_{|\psi|}^{q} 1\right) \circ f^{j q}} .
$$

The absolute convergence of this series is guaranteed by the condition $|z|$ $<\inf _{x} P_{|\psi|}^{q} 1(x)$. Moreover, using properties (3) and (4) listed in the Introduction, it is $x$
easy to verify the relation

$$
P_{\psi}^{q} e_{q, z}=z e_{q, z} .
$$

It remains to check that $e_{q, z}$ is continuous. This is obvious in the case where $\psi$ is a strictly positive function (so that $\chi_{q}(y)=1, \forall y \in[0,1]$ ). In the general case, let us note that $\prod_{j=0}^{l-1} \chi_{q} \circ f^{j q}$ is constant on the intervals of $\bigvee_{s=0}^{l q} f^{-s} \mathscr{A}$, where $\mathscr{A}$ denotes the Markov partition. Moreover, $h_{q} \circ f^{l q}$ has support inside the union of these open intervals. Hence $\left(\prod_{j=0}^{l-1} \chi_{q} \circ f^{j q}\right) \cdot\left(h_{q} \circ f^{l q}\right)$ is continuous. We then have a uniformly convergent series of continuous functions which is therefore continuous.

Remark. In the above construction it is implicit that $\psi$ is real-valued. One can also make things work for complex-valued weights. For the sake of simplicity, let us consider the case where $\psi$ is a strictly positive function. Thereafter we write $\psi=e^{u}$ and introduce a countable set of functions $\psi_{l}=e^{u+i(l+1) v}$, where $l=0,1,2, \ldots$ and $u, v \in C^{\alpha}$. 
We shall describe the essential spectrum of the operator

$$
\left(P_{\psi_{0}} h\right)(x)=\sum_{\substack{y \\ f(y)=x}} e^{u(y)+i v(y)} h(y) .
$$

Let us first note that

$$
\left|P_{\psi_{0}}^{q} 1(x)\right| \leqq P_{\psi}^{q} 1(x) .
$$

Let $h_{q} \in C^{0}[0,1]$ such that $P_{\psi}^{q} h_{q}=0$, as before. If $|z| \leqq \inf _{x} P_{\psi}^{q} 1(x)$, then

$$
\tilde{e}_{q, z}=\sum_{l=0}^{\infty} z^{l} \frac{\prod_{r=0}^{r=l} \prod_{s=0}^{s=q-1} e^{-i v \circ f^{s+r q}} \cdot\left(h_{q} \circ f^{l q}\right)}{\prod_{j=1}^{j=l}\left(P_{\psi}^{q} 1\right) \circ f^{j q}}
$$

is well defined and satisfies $P_{\psi_{0}}^{q} \tilde{e}_{q, z}=z \tilde{e}_{q, z}$.

Proof of the Theorem. From Lemma 2 and Lemma 4 we have

$$
\lim _{q \rightarrow \infty} \frac{1}{q} \log _{x} \inf _{|\psi|}^{q} 1(x)=F_{|\psi|} .
$$

Then, let us prove that for any number $\eta<e^{F_{|\psi|}}$ we can find infinitely many eigenvalues of $P_{\psi}$ on the circle of radius $\eta$. Indeed, from Lemma 5 we have that if $q$ is large enough, then any point of the circle of radius $\eta^{q}$ is an eigenvalue of $P_{\psi}^{q}$. Therefore one of the $q^{\text {th }}$ roots of this number must be an eigenvalue of $P_{\psi}$.

We can actually say more. Let $z$ be a number of modulus $\eta^{q}$. By modifying, if necessary, the function $r_{1}$ used in the definition of $h_{q}$, we can assume that the vectors

$$
e_{q, z}, P_{\psi} e_{q, z}, \ldots, P_{\psi}^{q-1} e_{q, z}
$$

are linearly independent. They generate an invariant finite dimensional subspace for $P_{\psi}$, and the corresponding eigen-equation is

$$
\lambda^{q}=z .
$$

Therefore, all of the $q^{\text {th }}$ roots of $z$ are eigenvalues of $P_{\psi}$. On the other hand, a sufficient condition for a complex number $\lambda$ to belong to the essential spectrum of a linear operator $T$, is that $\bigcup_{q \geq 1} \operatorname{ker}\left((T-\lambda I)^{q}\right)$ is of infinite dimension (see $[\mathrm{N}]$ for alternative definitions). Thus, passing to the limit $q \rightarrow \infty$ in the above setting, we conclude that the essential spectrum of $P_{\psi}$ contains the disk of radius $e^{F|\psi|}$.

From Lemma 2 it follows however that the spectral radius of $P_{\psi}$ is $\leqq e^{F|\psi|}$, and the theorem is proven.

Remarks. (1) Note that the eigenvalues constructed above, besides being of infinite multiplicity, are not isolated, and this is already a sufficient condition to be in the essential spectrum (we thank the referee for this remark).

(2) From the above discussion it follows that the essential spectrum of $P_{\psi}$ in $C^{0}$ coincides with its spectrum. This raises some interesting questions about the decay of correlation functions for observables living in this space.

(3) Theorem 1 implies in particular that the essential spectrum of the operator $P$ (that is $P_{\psi}$ with $\psi=\left|f^{\prime}\right|^{-1}$ ) in $C^{0}$ is the unit disk in the complex plane. 


\section{The Essential Spectrum in $C^{k}$}

6. Theorem. The essential spectrum of $P$ in $C^{k}$ is the disk of radius $e^{F(-k)}$.

Proof. The strategy is to reduce the $C^{k}$ problem to the $C^{0}$ one. Indeed, it is easy to prove recursively that

$$
(P h)^{(k)}(x)=\sum_{\substack{y \\ f(y)=x}} \frac{h^{(k)}(y)}{\left|f^{\prime}(y)\right|\left(f^{\prime}(y)\right)^{k}}+\sum_{\substack{y \\ f(y)=x}} \sum_{l=0}^{k-1} G_{l}(y) h^{(l)}(y),
$$

where the functions $G_{l}(y)$ are $C^{0}$ and $h^{(k)}$ denotes the $k^{\text {th }}$ derivative of $h$. Furthermore, since we have chosen $h$ in $C^{k}$, it is clear that the second term is compact in $C^{0}$. More precisely, we can write the above formula in the following way:

$$
(P h)^{(k)}(x)=P_{\psi} h^{(k)}(x)+\sum_{l=0}^{k-1} R_{l} K_{k-l} h^{(k)}(x)+\sum_{l=0}^{k-1} R_{l} h^{(l)}(0),
$$

where

$$
\psi=\left|f^{\prime}\right|^{-1}\left(f^{\prime}\right)^{-k} .
$$

The $R_{l}$ 's are bounded linear operators given by

$$
\left(R_{l} h\right)(x)=\sum_{\substack{y \\ f(y)=x}} G_{l}(y) h(y)
$$

and $K_{k-l}$ is a suitable composition of compact integral operators of the type

$$
h^{(k)}(x) \rightarrow \int_{0}^{x} h^{(k)}(u) d u-h^{(k-1)}(0) .
$$

In other terms, for any $k \geqq 1$, we have

$$
(P h)^{(k)}(x)=P_{\psi} h^{(k)}(x)+T_{k} h^{(k)}(x)+S_{k} h(x),
$$

where $T_{k}$ is a compact operator in $C^{0}$ (since it is obtained by composition of a bounded operator with a compact operator), and $S_{k}$ is of finite rank.

Now, since the essential spectrum is not modified by a compact perturbation (see [Ka], p. 244), the essential spectrum of $P$ in $C^{k}$ and of $P_{\psi}$ in $C^{0}$ (with $\psi$ as above) are identical. The final result follows from Theorem 1 and the expression (5) for $F(\beta)$.

Remarks. (1) Using a similar reasoning, one can prove that for any $k$ (even not integer), $e^{F(-k)}$ is an upper bound of the essential spectral radius.

(2) Since we have assumed $\left|f^{\prime}\right| \geqq \varrho>1$, then $F(-k) \leqq-k \log \varrho$. This means that the essential spectral radius tends to zero when $k \rightarrow \infty$. (For related results, see also [T, R3, P2]).

\section{References}

[Ba] Baladi, V.: Fonctions zêta, fonctions de corrélation et mesures d'équilibre pour quelques systèmes dynamiques non axiome $A$. Thesis presented at the Faculty of Sciences, University of Geneva, 1989

[BPPV] Benzi, R., Paladin, G., Parisi, G., Vulpiani, A.: J. Phys. A 17, 513 (1984) 
[BR] Bohr, T., Rand, D.: Physica D 25, 387 (1986)

[Bo] Bowen, R.: Equilibrium States and the Ergodic Theory of Anosov Diffeomorphisms. Lectures Notes in Mathematics, Vol. 470. Berlin, Heidelberg, New York: Springer 1975

[CL] Collet, P., Lesne, A.: J. Stat. Phys. 57, 967 (1989)

[Ec] Eckmann, J.-P.: Resonances in dynamical systems. In: IX $^{\text {th }}$ International Congress on Mathematical Physics. July 1988, Swansea, Wales, Simon, B., Truman, A., Davies, I.M. (eds.). Bristol, New York: Adam Hilger 1989

[El] Ellis, R.: Entropy, Large Deviations and Statistical Mechanics. Berlin, Heidelberg, New York: Springer 1985

[GH] Guckenheimer, J., Holmes, P.: Nonlinear Oscillations, Dynamical Systems and Bifurcations of Vector Fields. Berlin, Heidelberg, New York: Springer 1983

[H] Haydn, N.T.A.: Meromorphic extension of the zeta function for Axiom A flows, Preprint (1989). To appear in Ergod. Th. Dynam. Sys.

[K1] Keller, G.: On the rate of convergence to equilibrium in one-dimensional systems. Commun. Math. Phys. 96, 181-193 (1984)

[K2] Keller, G.: Markov extensions, zeta-functions, and Fredholm theory for piecewise invertible dynamical systems. Trans. Am. Math. Soc. 314, 433-499 (1989)

[Ka] Kato, T.: Perturbation theory of linear operators, II Ed. Berlin, Heidelberg, New York: Springer 1976

[M] Mañe, R.: Ergodic theory and differentiable dynamics. Berlin, Heidelberg, New York: Springer 1987

[N] Nussbaum, R.D.: The radius of the essential spectrum. Duke Math. J. 37, 473-478 (1970)

[P1] Pollicott, M.: Meromorphic extensions of generalized zeta functions. Invent. Math. 85, 147-164 (1986)

[P2] Pollicott, M.: The differential zeta function for Axiom A attractors. Ann. Math. 131, 331-354 (1990)

[R1] Ruelle, D.: Thermodynamic formalism. Reading, MA: Addison-Wesley 1978

[R2] Ruelle, D.: Locating resonances for Axiom A dynamical systems. J. Stat. Phys. 44, 281-292 (1987)

[R3] Ruelle, D.: The thermodynamic formalism for expanding maps. Commun. Math. Phys. 125, 239-262 (1989)

[R4] Ruelle, D.: An extension of the theory of Fredholm determinants. Preprint IHES/P/89/38 (1989)

[S] Sinai, Ya.G.: Gibbs measures in ergodic theory. Russ. Math. Surv. 166, 21-69 (1972)

[T] Tangerman, F.: Meromorphic continuation of Ruelle zeta functions. Boston University thesis (1986)

Communicated by J.-P. Eckmann 
